\title{
Hidrógeno verde en Argentina ¿un nuevo orden extractivo?
}

\author{
Martín Ariel Kazimierski ${ }^{1}$ \\ Universidad de Buenos Aires \\ Consejo Nacional de Investigaciones Científicas y Técnicas \\ @ [ martin.kazimierski@gmail.com ]
}

RECIBIDO 02-03-2021

ACEPTADO 30-07-2021

Cita sugerida: Kazimierski, M. A. (2021). Hidrógeno verde en Argentina iun nuevo orden extractivo? Revista Huellas, Volumen 25, № 2, Instituto de Geografía, EdUNLPam: Santa Rosa. Recuperado a partir de: http://cerac.unlpam. edu.ar/index.php/huellas

DOI: http://dx.doi.org/10.19137/huellas-2021-2521

\begin{abstract}
Resumen
Las tendencias globales que apuntan a una incorporación masiva de las energías renovables, apuntan también a nuevas tecnologías de almacenamiento energético. La producción de hidrógeno verde a través de la electrólisis del agua se erige en este contexto como una de las alternativas más prometedoras, formando parte de múltiples planes nacionales. No obstante, los estudios técnicos y ambientales, así como los marcos normativos que regulan esta incipiente actividad, son todavía escasos. Teniendo en cuenta su potencial en la región, particularmente en Argentina, el objetivo de este trabajo consiste en identificar y describir los desafíos ambientales y regulatorios que plantea la industria del hidrógeno verde en el país. Partimos de una descripción del método de obtención y las prácticas usuales de manejo a nivel internacional, para luego abordar el caso argentino y hacer un análisis crítico con los principales lineamientos ambientales a tener en cuenta.
\end{abstract}

Palabras clave: hidrógeno verde; energía renovable; electrólisis; impacto ambiental

Green hydrogen in Argentina, a new extractive order?

\begin{abstract}
Global trends aiming at a massive incorporation of renewable energies also point to new energy storage technologies. The production of green hydrogen through the electrolysis of water stands out in this context as one of the most promising alternatives, partaking in multiple national plans. However, technical and environmental studies, as well as the normative frameworks that regulate this emergent activity, are still scarce. Taking into account its potential in the
\end{abstract}


region, particularly in Argentina, the aim of this work is to identify and describe the environmental and regulatory challenges posed by the green hydrogen industry in the country. We start from a description of the extraction method and the usual management practices at the international level, to then address the Argentine case and make a critical analysis with the main environmental guidelines to be taken into account.

Keywords: green hydrogen; renewable energy; electrolysis; environmental impact

\title{
Hidrogênio verde na Argentina, uma nova ordem extrativa?
}

\begin{abstract}
Resumo
As tendências globais que assinalam uma incorporação massiva das energias renováveis, apontam também a novas tecnologias de armazenamento energético. A produção de hidrogênio verde a través da eletrólise da água se erige neste contexto como uma das alternativas mais prometedoras, formando parte dos múltiplos planos nacionais. Não obstante, os estudos técnicos e ambientais, assim como os marcos normativos que regulam esta incipiente atividade são ainda escassos. Levando em consideração seu potencial na região, particularmente na Argentina, o objeto deste trabalho é identificar e descrever os desafios ambientais e de regulamentação que propõe a indústria do hidrogênio verde no país. Partimos de uma descrição do método de obtenção e as práticas usuais de gestão a nível internacional para logo abordar o caso argentino e fazer uma análise crítica com os principais alinhamentos ambientais a serem considerados.
\end{abstract}

Palavras-chave: Hidrogênio verde; energia renovável; eletrolise; impacto ambiental

\section{Introducción}

En los últimos años, tanto el mercado energético como las políticas desplegadas desde los organismos nacionales e intergubernamentales, impulsan una decidida transición hacia sistemas energéticos más "verdes". Los principios rectores se sostienen en una importante electrificación de la matriz energética, con la incorporación masiva de nuevas fuentes renovables disponibles localmente; y el despliegue de toda una infraestructura de acumuladores y vehículos eléctricos. Para el año 2020, la tecnología renovable -fundamentalmente eólica y solar- ha escalado al 14\% de la oferta energética mundial, y se espera un crecimiento sustancial de hasta el 70\% para el 2040 (IEA, 2019). No obstante, el carácter intermitente de estas fuentes de energías y el hecho de que la electricidad no puede 
ser acumulada sino es a través de procesos de transformación, posiciona al almacenamiento energético como la piedra angular de esta transición, incluyéndose como parte vital de los planes de energía en diferentes países.

Una tecnología de almacenamiento que se espera que desempeñe un papel protagónico en el mediano plazo es el hidrógeno. Este es el elemento químico más abundante del universo y el tercero más abundante en la Tierra, y aunque se denomina como el combustible del futuro, en realidad no es una fuente primaria de energía, sino un vector energético, es decir, un portador de energía. La conversión de electricidad en hidrógeno para el almacenamiento y el transporte a largas distancias se perfila revolucionario, augurando lo que se conoce como una nueva economía del hidrógeno, esto es, un modelo alternativo al uso de combustibles fósiles (Rifkin, 2002).

El hidrógeno no se encuentra libre en la naturaleza, sino combinado con otros elementos como el oxígeno, el carbono y el nitrógeno (Gutiérrez, 2005). Está presente en recursos como el agua, hidrocarburos, biomasa, sulfuro de hidrógeno y desechos antropogénicos (aguas residuales, desechos agrícolas, entre otros residuos que generan gases de vertedero por degradación anaerobia). Actualmente, su demanda mundial comprende más de 70 millones de toneladas al año, casi el $80 \%$ derivado del gas natural y un $20 \%$ del carbón, lo cual provoca la emisión de ingentes cantidades de dióxido de carbono por año². No obstante, debido a los compromisos asumidos en el Acuerdo de París en 2015 para reducir las emisiones de Gases de Efecto Invernadero (GEI), así como también a la baja en el costo de generación de energía renovable, la obtención de hidrógeno por medio de la electrólisis del agua se ha erigido rápidamente como alternativa verde. Este método consiste en la descomposición de las moléculas de agua $(\mathrm{H} 2 \mathrm{O})$ en oxígeno $(\mathrm{O} 2)$ e hidrógeno $(\mathrm{H} 2)$, a través de una corriente eléctrica que sería provista por una fuente renovable, según la disponibilidad del recurso (Morante et al, 2020).

En conjunto, las energías renovables y la tecnología del hidrógeno enarbolan la bandera de un nuevo capitalismo verde o sostenible, que aspira a superar las contradicciones inherentes del capitalismo fósil y su relación con la naturaleza. Particularmente, nos permiten debatir las ideas de O' Connor (2000) sobre un capitalismo que destruye sus propias condiciones de producción y las de Harvey (2004, p. 244) sobre las oportunidades para que un "capitalismo del desastre" obtenga mayores beneficios. La tecnología del hidrógeno se perfila como una alternativa seria de transformar la relación metabólica con la naturaleza, así como también aspira a transformar las prácticas económicas de los Estados y las corporaciones. Anuncios oficiales recopilados por la Agencia Internacional de Energía acu- 
mulan una inversión de USD 108 mil millones en 2021 para paquetes de desarrollo verde, de los cuales USD 20 mil millones serían sólo para el hidrógeno (IEA, 2019). La Agencia estima que, para descarbonizar el sistema energético mundial, se requerirá incrementar para el año 2050 la producción de 520 megatoneladas (Mt) anuales, de las cuales $300 \mathrm{Mt}$ corresponderían al hidrógeno verde (IEA, 2021).

Seis países europeos, además de Rusia, ya anunciaron una "Hoja de Ruta" con la incorporación de energías renovables e hidrógeno verde a sus matrices energéticas, en línea con los planes de recuperación de la agenda post Covid-19. La región sudamericana no se queda atrás. Chile, el laboratorio por excelencia del capital para la experimentación en nuevas tecnologías energéticas, anunció su Estrategia Nacional de Hidrógeno Verde con tres objetivos principales: producir el hidrógeno verde más barato del planeta para 2030; estar entre los 3 principales exportadores para 2040; y contar con 5 GW de capacidad de electrólisis en desarrollo al 2025 (Ministerio de Energía, 2020). La producción alcanzaría valores de 25 millones de toneladas al año, con ingresos anuales superiores a los USD 30 mil millones. Planes similares también se han oficializado en Costa Rica, Colombia, Uruguay, Brasil y Paraguay, acompañadas por organizaciones internacionales involucradas directamente en la promoción del capitalismo verde como la Agencia Internacional de Energía, el BID, el Banco Mundial y GIZ (IEA, 2021). Junto a esto, también se han venido instalando o proyectando nueva infraestructura con la finalidad de incrementar la producción de hidrógeno en la región, principalmente con fines de exportación n a Estados Unidos, Europa y Japón. El Estado argentino también tiene previsto apuntalar esta actividad. Durante el gobierno de Mauricio Macri (2015-2019) se hizo un primer intento a partir de una iniciativa de la automotriz Toyota, como un plan para fomentar el trabajo en conjunto entre Argentina y Japón; por su parte, el gobierno de Alberto Fernández (20192023) oficializó la creación de un consorcio de empresas para convertir al país en un productor a gran escala, proyecto que tiene como protagonista a YPF y sus filiales, y que va de la mano de la actualización del Régimen Legal de Promoción del Hidrógeno (Sanguinetti, 2020).

En vistas del potencial que tiene esta tecnología en Argentina, y en toda región sudamericana, el objetivo de este trabajo consiste en identificar y describir los desafíos regulatorios y ambientales que plantea la industria del hidrógeno verde en el país. La pregunta medular que atraviesa el estudio es iestamos frente a un nuevo orden extractivo? Una anticipación de sentido es que existe la posibilidad de que este incipiente mercado -que mercantiliza un recurso vital como el agua-, se traduzca en una nueva política de desposesión (Harvey, 2004), relegando a la región a su papel más tradicional de productores de materias primas y extractores de 
recursos para el mundo industrializado, dejando tras de sí las externalidades negativas del nuevo "extractivismo verde". Los interrogantes que se levantan en este escenario son múltiples: ¿cuán verde es el hidrógeno renovable?, ¿existen externalidades negativas asociadas a su producción?, ¿qué dice la experiencia internacional y los informes oficiales respecto a la dimensión ambiental?

Pese a que la "sostenibilidad" es una cuestión ideológica y política, antes tecnológica (O' Connor, 2000), este trabajo representa una primera aproximación de carácter exclusivamente técnica sobre la producción de hidrógeno verde, prescindiendo de las etapas posteriores (transporte, almacenamiento y dispensación), así como de los aspectos ambientales que remiten estrictamente al desarrollo de las energías renovables. Partiremos de una descripción del método de obtención y las prácticas usuales de manejo a nivel internacional; seguidamente, haremos foco en la coyuntura nacional argentina y los antecedentes en cuanto a la regulación ambiental y sectorial; para luego finalizar con un análisis crítico sobre los aspectos ambientales que podrían conllevar esta actividad y que necesariamente deben incluirse en los estudios ambientales.

\section{Método de producción del hidrógeno verde}

A pesar de la connotación futurista que se teje alrededor del hidrógeno, las primeras aplicaciones con este elemento se remontan a la experimentación con globos de finales del siglo XVI; evolucionando luego hacia dirigibles de grandes dimensiones en el último siglo. El incendio del zepelín alemán Hindenburg en 1937 y el ascenso indiscutible de los combustibles fósiles, mermaron la popularidad de este vector energético, reapareciendo con nuevos usos tras la crisis del petróleo de 1973, fundamentalmente, la pila de combustible y los motores de combustión interna que queman hidrógeno para mover vehículos y cohetes. Con ellos, una centena de métodos para su producción también fueron desplegados. Winter (1988) los divide en cuatro categorías básicas: biológicos, químicos, electroquímicos y térmicos. Por su parte, Dincer (2012) los clasifica en seis: electroquímicos -a través de una corriente eléctrica que descompone el agua en $\mathrm{O}_{2}$ y $\mathrm{H}_{2}$-; termoquímicos -reacciones químicas impulsadas por energía de ignición o calor-; fotoquímicos -electrólisis originada por energía foto-catalítica o foto-electroquímica-; radio-químicos -descomposición del agua por radiación nuclear-; bioquímica-obtención de hidrógeno por conversión de biomasa-; e híbridos.

Hoy en día, el método más común es el denominado hidrógeno gris. Este se basa en el reformado con vapor de gas natural, empero, al igual que para otros combustibles fósiles, sigue produciendo $\mathrm{CO}_{2}$, principal compo- 
nente de los GEl. Como alternativa, se ha propuesto el hidrógeno azul, que también se obtiene del gas natural pero que incluye el uso de la tecnología de captura y almacenamiento de carbono (CAC) en el proceso. El hidrógeno verde, por su parte, es el que se proyecta con mayor grado de desarrollo, y aunque actualmente presume del $4 \%$ de la producción mundial de hidrógeno, se espera que aumente conforme se reduzcan sus costos (ver Figura $\mathrm{N}^{\circ} 1$ ). Según un informe de la consultora IHS Markit (2020), los costes de producción de hidrógeno verde han caído un $40 \%$ en solo cinco años, desde 2015, y está previsto que caigan otros 40 puntos hacia 2025.

Figura $\mathbf{N}^{\circ} 1$. Tipos de hidrógeno en función de la procedencia del recurso

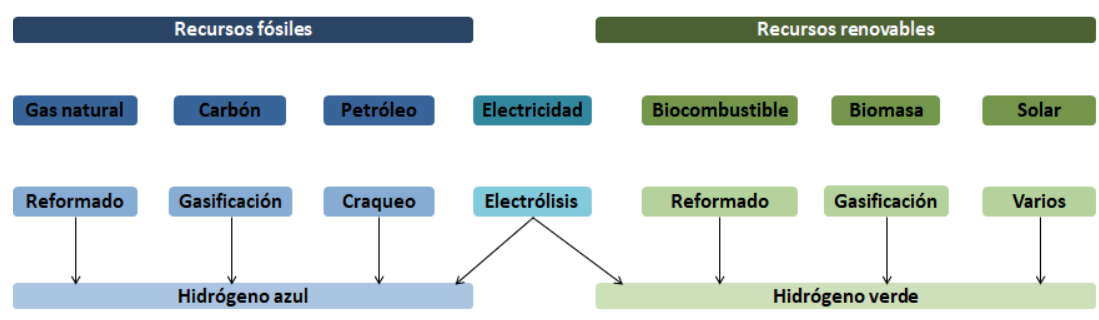

Fuente: elaboración propia en base a Morante et al (2020).

El principal método de obtención de hidrógeno verde es a través de la electrólisis del agua, que consta de dos insumos básicos: agua y electricidad. La electricidad puede ser suministrada por la red eléctrica o por un generador propio de la planta -aunque para que se considere verde debe estar conectada a una fuente renovable-, mientras que el agua puede ser provista por una instalación de tratamiento cercana, o directamente de una fuente natural superficial o subterránea. Tal como se observa en la Figura $\mathrm{N}^{\circ} 2$, el proceso consta de tres etapas: (1) el acondicionamiento del agua; (2) electrólisis; y (3) el acondicionamiento de gases; obteniendo, de esta manera, oxígeno como residuo e hidrógeno como producto.

Figura $\mathbf{N}^{\circ 2}$. Producción de hidrógeno verde

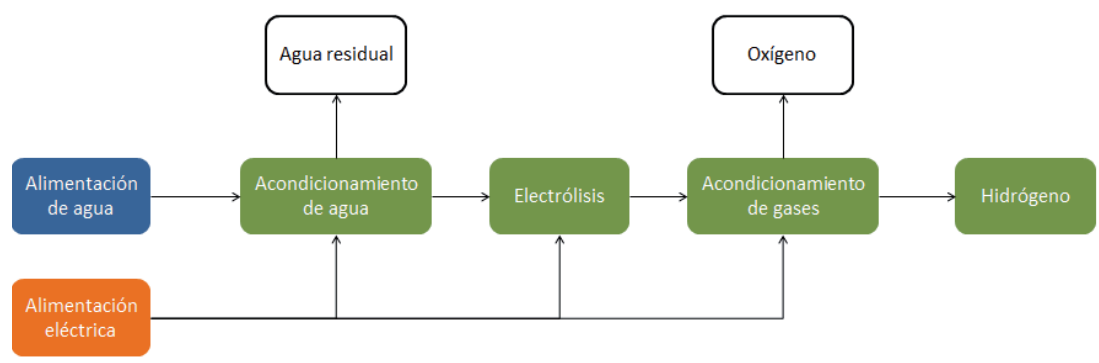

Fuente: elaboración propia en base a Inodú (2020). 
El sistema de acondicionamiento de agua tiene por objetivo producir agua de alta pureza a partir de la extracción de sus sales disueltas. De acuerdo con Inodú (2020), documento oficial del Ministerio de Energía chileno, el proceso involucra descalcificación, osmosis reversa, membrana de desgasificación y electrodesionización, generando aguas residuales en rangos de $30 \%$ a $43 \%$ con una alta conductividad. Para este informe, estas aguas pueden ser descargadas directamente al sistema de alcantarillado en bajos volúmenes, cumpliendo los requerimientos establecidos.

En cuanto al proceso de electrólisis, este puede llevarse a cabo a través de tres tecnologías principales, definidas por la Agencia Internacional de Energía (IEA, 2019): (1) Electrólisis alcalina (AEL); (2) Electrólisis con membrana de intercambio de protones (PEM); y (3) Electrólisis de alta temperatura (SOEC). Los electrolizadores alcalinos representan la tecnología de electrólisis de agua más desarrollada y de menor coste de inversión. Sin embargo, debido a las características del electrolito utilizado, esta produce bajas densidades de corriente, lo que se traduce en bajas producciones y sistemas de purificación complejos. Al contrario, el método de electrólisis con PEM es más eficiente, aunque los costes de inversión son más altos y depende en gran medida de materias primas como los metales preciosos. Por último, el método SOEC es el que presenta mejores eficiencias, aunque su aplicación es todavía incipiente (Morante et al, 2020).

La etapa final es el de acondicionamiento de los gases de salida. Estos primero pasan por un sistema de separación de gases del electrolito, donde el electrolito se recircula, el oxígeno se libera a la atmósfera y el hidrógeno se dirige a la etapa de purificación y secado. Allí, un sistema de filtros de coalescencia permite eliminar el electrolito arrastrado por las corrientes gaseosas y los filtros desecantes eliminar la humedad restante. Debido a que en la electrólisis se busca obtener hidrógeno con la mayor pureza posible, el gas pasa por un reactor de desoxigenación que elimina las posibles impurezas de oxígeno arrastradas. Nuevamente, el oxígeno residual es liberado a la atmósfera, mientras que el hidrógeno de alta pureza es almacenado en recipientes de baja presión, de acuerdo con las condiciones específicas de salida del sistema, para posteriormente ser tratado de acuerdo con sus requerimientos de uso.

En cuanto a las implicancias ambientales de este proceso, las incipientes experiencias a nivel internacional destacan el leve impacto de las centrales productoras de hidrógeno en sus proximidades. El informe Inodú (2020), desarrollado por el Ministerio de Energía chileno y Deutsche Gesellschaft für Internationale Zusammenarbeit (GIZ) GmbH, ofrece una síntesis con dos experiencias en Alemania, el país con el plan más ambicioso para el hidrógeno verde. El Procedimiento de Aprobación de la planta Energiepark Mainz -la instalación de mayor capacidad a nivel mundial en 
funcionamiento a partir de la tecnología PEM-, destaca que la central no genera emisiones de gases contaminantes, ni residuos sólidos; tampoco produce olores o ruidos molestos que superen los niveles permitidos por la legislación alemana. Por último, destaca que el proyecto carece de un impacto ambiental adverso significativo que deba tenerse en cuenta en virtud de la legislación vigente; por lo tanto, no hay obligación de realizar un estudio del impacto ambiental (Grün-und Umweltamt Mainz, 2019 en Inodú, 2020). Situación análoga se presenta en el caso de la Central Power-to-Gas Grenzach-Wyhlen, una planta de electrólisis alcalina de 1 MW de capacidad acoplada a una planta piloto de $300 \mathrm{~kW}$, que alcanza una producción electrolítica de hasta 300 Nm3/h (normal metros cúbicos por hora) (Regierungspräsidium Freiburg, 2018 en Inodú, 2020).

Estas experiencias dejan en claro que para gran parte de los informes que abordan la cuestión ambiental, la peligrosidad del hidrógeno se circunscribe exclusivamente a su alta inflamabilidad, por lo que el riesgo no está determinado por la capacidad de producción, sino por la cantidad que se tenga almacenada. No se mencionan aspectos que den cuenta de los impactos causados por la disposición de grandes masas de agua de alta conductividad, ni las consecuencias que puede traer la demanda de ingentes volúmenes agua sobre el entorno y el ecosistema.

\section{El marco legal argentino}

Las iniciativas relacionadas con el hidrógeno verde presentan desafíos regulatorios importantes, sobre todo en Sudamérica, ya que es inexistente, o bien insuficiente, genérica y desactualizada. En este sentido, aquella normativa vigente simplemente regula el hidrógeno como una sustancia peligrosa, sin reconocer toda su cadena de valor, o sin considerar una regulación específica para el emplazamiento de esta actividad. Argentina es el único país que tiene una legislación específica, aunque sólo se refiere a él en términos generales a su desarrollo y aplicación como combustible, sin distinguir los distintos tipos (o colores) de hidrógeno, menos aún los lineamientos y requisitos para su producción.

El régimen actual se remonta al año 2006, cuando fue promulgada la Ley N²6.123 de "Régimen para el desarrollo de la tecnología, producción, uso y aplicaciones del hidrógeno como combustible y vector de energía". Allí se declara de interés nacional el desarrollo de esta tecnología, se promueve la investigación, producción y aplicación, previendo además la redacción de un Plan Nacional de Hidrógeno (PNH), que tendría lugar recién en 2013. El Plan postula específicamente 24 grandes emprendimientos a implementar en distintos períodos: siete propuestas están contempladas para lo inmediato, ocho para el mediano plazo (incluyendo la 
posible inyección de hidrógeno en las redes de gasoductos) y otras siete para el largo plazo (vinculadas con los vehículos de transporte, colectivos, la formación de recursos humanos y la producción de hidrógeno a partir de la biomasa) (Petroquímica, 2020). Pese a ello, hasta hoy no han existido incentivos verdaderamente efectivos por parte del Estado para fomentar el desarrollo de esta industria.

Los discursos y fuentes oficiales se remiten a las "excelentes" condiciones naturales que ofrece el país para la generación de hidrógeno tanto verde como azul (Sanguinetti, 2020), fundamentalmente porque el sistema energético argentino ha profundizado su inserción en el mercado gasífero, con la explotación del yacimiento no convencional Vaca Muerta, y en el mercado de energías alternativas, con la sanción de la Ley №27.191 de Régimen de Fomento Nacional para el Uso de Fuentes Renovables en 2015 -que modifica la Ley №26.190 de 2006-. Este establece el objetivo de alcanzar una participación del $20 \%$ al 2025, para lo cual se han llevado a cabo rondas licitatorias que adjudicaron más de $6000 \mathrm{MW}$ de potencia $-15 \%$ del total instalado- en apenas tres años (2016-2019).

En este contexto, el desarrollo del hidrógeno verde se presenta cada vez más como una oportunidad concreta para la ciencia y la industria, así como para las fuerzas productivas que buscan convertir al país en un mercado exportador. La propuesta del directorio de YPF para que una de sus filiales lidere este proceso -aprovechando la escala de negocios que posee la petrolera en el mercado de renovables-, sitúa a la región patagónica como uno de los lugares privilegiados en donde se puede alcanzar la mayor escala de producción (Sanguinetti, 2020). Esto se debe a la disponibilidad de agua y al potencial del recurso eólico, que incluye vientos de calidad internacional de hasta 9-12 m/s. Justamente allí, en las afueras de la localidad de Comodoro Rivadavia -provincia de Chubut-, se ubica la única planta operativa para la producción de hidrógeno por medio del proceso de electrólisis, con una capacidad de producción anual de $850.000 \mathrm{Nm}^{3}$. La planta, propiedad de Hychico -sociedad controlada por Compañías Asociadas Petroleras S.A. (CAPSA)-, es alimentada por el Parque Eólico Diadema, propiedad de la misma firma, con 6,3 MW de potencia.

En cuanto a Estudios de Impacto Ambiental (EIA) asociados a la planta de Hychico, sólo se ha encontrado un único antecedente que se circunscribe a una serie de estudios geológicos y relevamientos de instalaciones con el fin de analizar la factibilidad del almacenamiento subterráneo de hidrógeno en un reservorio depletado de petróleo y gas ${ }^{3}$. Para este proyecto piloto, $\mathrm{Hy}$ chico presentó en 2010 el estudio correspondiente y realizó una audiencia pública coordinada por el Ministerio de Ambiente y Control de Desarrollo Sustentable de la Provincia de Chubut, logrando así su aprobación en mayo 
de 2014. Un año más tarde, se construyó un ducto de 2,3km vinculando la planta con el pozo F-160, donde se realiza el proyecto piloto.

En vistas de la ausencia de normativa, informes y estudios concretos que aborden las implicancias ambientales de la producción de hidrógeno a través de la electrólisis del agua, a continuación, se buscará aportar algunos lineamientos básicos que sirvan como disparadores para pensar esta temática.

\section{Lineamientos para pensar la dimensión ambiental}

El presente apartado tiene como objetivo identificar y evaluar los posibles impactos ambientales que se podrían generar por la masificación de la tecnología del hidrógeno verde en el país. Por impacto ambiental nos referimos a una alteración favorable o desfavorable en el medio, o en alguno de sus componentes, como consecuencia de una actividad particular. Siguiendo a Conesa et al (2010), debemos tener en cuenta tanto los factores ambientales -elementos, cualidades y procesos del entorno- que pueden ser afectados, así como el conocimiento de las condiciones ambientales del área de estudio, esto es, la Línea de Base.

Para el caso, las principales reglamentaciones a considerar se circunscriben a la Ley №26.123: Régimen para el desarrollo de la tecnología, producción, uso y aplicaciones del hidrógeno como combustible y vector de energía; Ley No25.675: Ley General del Ambiente; Ley No27.191: Régimen de Fomento Nacional para el Uso de Fuentes Renovables; Ley No27.270; Adhesión al Acuerdo de París; además de las diferentes disposiciones provinciales. En cuanto a la identificación de acciones impactantes, un estudio realizado por Rodríguez et al (2009) elaboró una matriz para una hipotética planta de producción de hidrógeno en la provincia de Córdoba alimentada por energía eólica. Este tuvo en cuenta las acciones en las distintas fases del proyecto (construcción-operación-abandono) para cada uno de los factores afectados, ponderando su:

- intensidad: la medida del cambio cualitativo de un parámetro ambiental, provocado por una acción (baja - media - alta - muy alta - total)

- extensión: acción del área de estudio que será potencialmente afectada por el impacto (puntual - parcial - amplio o extenso - total - crítico)

- efecto: manifestación del efecto sobre un factor ambiental (directo - indirecto)

- persistencia: tiempo de permanencia del efecto sobre un factor ambiental (momentáneo - transitorio - persistente - permanente) 
- reversibilidad: posibilidad de que el factor ambiental afectado regrese a su estado natural inicial por medios naturales (corto plazo - mediano - largo - irreversible).

El estudio identificó ocho acciones en la etapa de operación de la planta: (1) presencia física del parque eólico; (2) movimiento de las palas; (3) presencia del tendido eléctrico; (4) generación de energía; (5) uso del recurso agua para el funcionamiento de los electrolizadores; (6) mantenimiento de aerogeneradores; (7) generación de residuos; y (8) uso de vehículos. Entre los factores impactados, destaca: (a) la calidad del aire y (b) el ruido; (c) el consumo y (d) la calidad del agua; (e) la calidad del suelo; (f) la calidad del paisaje; (g) el desplazamiento de fauna; (h) la demanda de empleo; y (i) la afectación a la actividad agropecuaria. La mayor parte de ellos tienen al parque eólico como acción impactante, mientras que los electrolizadores influyen sólo en el consumo de agua y en la calidad del aire. Sin dar precisiones respecto a los guarismos construidos, el estudio concluye que, con una adecuada planificación de las acciones involucradas en las diferentes etapas, el proyecto puede ser ambientalmente viable.

Más contundente es el caso chileno. El informe Inodú (2020, p. 31) afirma que "no identifica riesgos ambientales en la producción de hidrógeno verde, ni en toda la cadena de valor". Destaca que el proceso de electrólisis es silencioso y no emite vibraciones; tampoco generan emisiones de NOx, CO, partículas o Carbono Orgánico Total, por lo que no es necesario aplicar medidas preventivas en este sentido. Al contrario, si se advierte sobre los vertidos de agua residual procedente de la depuración de agua utilizada para el proceso de electrólisis, aunque se asegura que pueden ser descargadas directamente al sistema de alcantarillado, a pesar de contar con un mayor grado de conductividad. En cuanto a las afecciones sobre la fauna y la flora, estas sólo son contempladas en relación a la instalación de energía renovable (eólica o solar) asociada a la planta.

Ahora bien, tal como hemos visto, la materia prima para la producción de hidrógeno verde es el agua. Los informes señalan que por cada tonelada de hidrógeno se utilizan alrededor de 10 toneladas de agua y se producen 8 toneladas de oxígeno (Asociación Chilena de Hidrógeno, 2020). Pero si tenemos en cuenta que el agua que el electrolizador descompone necesita ser purificada, son dos toneladas de agua impura para producir una tonelada de agua purificada. En otras palabras, una tonelada de hidrógeno en realidad no necesita 10 toneladas de agua, sino el doble de ello. Para Webber (2007), estos guarismos se asemejan a los requisitos de agua para refinar petróleo, por lo que las diferencias en los impactos directos del uso de agua serían mínimas. Para la Asociación Chilena de Hidrógeno (2020), el proceso requeriría apenas $0,6 \%$ de uso consuntivo del agua a 
nivel nacional, suficiente para producir el equivalente a todo el diésel, gas natural, gas licuado y gasolina consumido en Chile. Teniendo en cuenta estas consideraciones, y siguiendo a Conesa et al (2010), podemos decir que los estudios ponderan una intensidad del impacto baja, y una extensión de tipo puntual, es decir, de efecto localizado en la planta.

Sin embargo, la producción de hidrógeno verde con electrolizadores a gran escala naturalmente pone un límite a la elección de lugares adecuados. En principio, debería ubicarse en un área con una amplia disponibilidad del recurso hídrico, pero, además, para ser "ecológico", también tendría que estar cerca de un parque solar o eólico. Estos, lógicamente, no pueden ser construidos en cualquier lugar; los parques solares son más rentables en lugares con alta radiación solar, y los parques eólicos funcionan mejor en lugares donde hay suficiente viento. En general, estos lugares no se ubican cerca de vías fluviales (excepto los vientos marinos u offshore), sino que, en Argentina, el recurso solar de mejor calidad se ubica en las regiones más áridas de la región Noroeste, y el eólico en la estepa patagónica, región con escasas precipitaciones. Este dato no es menor, ya que según la consultora IHS Markit (2020), la cartera de proyectos de fábricas de hidrógeno verde a desarrollar en el decenio 2020-2030 supera los 23.000 MW de capacidad, más de 280 veces la capacidad actual ( $82 \mathrm{MW}$ ), proyectándose como el subsector de mayor consumo eléctrico del mundo.

La proliferación de parques de generación de energía renovable y el consumo de agua tendería a acelerarse, acentuando la especulación sobre tierras y territorios que ya había comenzado con otros proyectos eléctricos anteriores, coincidiendo en Argentina con áreas que podrían ser particularmente sensibles en sus balances hídricos. Teniendo en cuenta estos factores ambientales, la intensidad y la extensión del impacto podrían verse alterados, alcanzando grados de afectación significativos para el conjunto del sistema hidrogeológico. El hecho de que este tipo de impacto sea acumulativo no es menor. El Manual de Buena Práctica. Evaluación y Gestión de Impactos Acumulativos: Guía para el Sector Privado en Mercados Emergentes, desarrollado por la International Finance Corporation (IFC, 2015), destaca que una acción generada en un lugar o momento específico puede repercutir en un área geográfica y en un tiempo diferente, por lo que este tipo de impactos trascienden el concepto tradicional de "área de influencia directa", y recalcan la necesidad de analizar los efectos indirectos y acumulativos, así como la necesidad de expandir los límites geográficos de la evaluación de impacto y/o el marco temporal usado para el análisis.

Es claro que la aplicación de esta técnica es todavía incipiente, y su producción mundial comprende una porción menor, por lo que los efectos ambientales de su práctica a gran escala son aún inciertos. No obstante, su presencia cada vez más fuerte en los discursos y planes de energía ofi- 
ciales, especialmente en la región sudamericana, nos exige adelantarnos a los hechos y prepararnos para los desafíos que plantea el paradigma energético porvenir. Es necesario conocer profundamente los factores críticos del hidrógeno verde, más aún teniendo en cuenta que existen vacíos de información, o se encuentra fraccionada y de difícil acceso.

Por ello, resulta imperioso que la actualización del Régimen Legal de Promoción del Hidrógeno, impulsado por el actual gobierno, contemple la realización de una Evaluación Ambiental Estratégica (EAE) que contribuya a identificar los impactos ambientales y evaluar los efectos acumulativos que puedan surgir de una política o plan nacional sostenidos para esta actividad. Siguiendo a Herrera y Bonilla (2009), una EAE no busca anticipar y evaluar los efectos asociados a una actuación como lo haría una EIA, sino desarrollar marcos de planificación que tiendan a integrar los criterios ambientales a políticas, planes y programas (PPP), y de ese modo mejorar el perfil ambiental del marco en el que luego se desarrollen proyectos concretos. Su implementación típicamente incorpora una variedad de métodos entre los que se incluyen tanto la dependencia como la evaluación de impacto sobre los servicios ecosistémicos, el análisis, diagnóstico e identificación de escenarios actuales y futuros, y las políticas y otras intervenciones que buscan su sostenibilidad.

El reconocimiento del agua como un servicio ambiental clave para las dimensiones estructural, funcional y cultural del ser humano debe ser un elemento de análisis integral y de aplicación efectiva a todos los PPP que anteceden a cualquier tipo de proyecto extractivo. Sólo así estaríamos frente a alternativas verdaderamente ecológicas.

\section{Conclusiones}

La lucha del capitalismo para mejorar sus propias condiciones ecológicas está vigente y se agudiza cada vez más. Por ello, en los últimos años, las energías renovables y la tecnología del hidrógeno se han convertido en alternativas cada vez más serias para un paradigma post-fósil. La posibilidad de acoplar estas dos técnicas a través del proceso de electrólisis de agua potencia el imaginario de un crecimiento económico perpetuo y permite vislumbrar aquello que Harvey (2004, p. 244) expone de manera contundente: "es perfectamente posible que el capital continúe circulando y acumulándose en medio de catástrofes medioambientales". Por caso, no es casualidad que en los 230 proyectos operativos en Europa al 2019, el $80 \%$ involucre a empresas de combustibles fósiles (IEA, 2019).

Pese a que sus costos son aún superiores a los de reformado de vapor de los combustibles, las economías de escala y los avances tecnológicos, sumados a los esfuerzos internacionales por reducir las emisiones de car- 
bono, permiten predecir un aumento significativo en su producción en los próximos años. Estas perspectivas exigen una mirada crítica, pues aquello que se presenta como verde necesariamente oculta la naturaleza entrópica de los procesos productivos. En este sentido, el hidrógeno verde puede ser la llave para un futuro más sustentable, o bien, una nueva forma de cercamiento de los bienes comunes, de mercantilizar, privatizar e incorporar cada vez más aspectos de nuestro mundo a los circuitos capitalistas, y de ampliar las disparidades en el bienestar económico entre los países del sur y los del norte.

A través del análisis de diferentes fuentes, el trabajo ha identificado los núcleos básicos que disparan el debate ambiental del hidrógeno verde en la región. Lo más sobresaliente es que de las tres tipologías de proyectos definidas en Inodú (2020): producción, almacenamiento y transporte, el informe enfatiza los riesgos de las últimas dos, es decir, el riesgo no estaría determinado por la capacidad de producción, sino por la cantidad almacenada y transportada. En esta línea, el estudio de Rodríguez et al (2009) ofrece un desglose de los factores ambientales y las actividades potencialmente riesgosas para la instalación de una central en la provincia de Córdoba, pero cuyo análisis se centra en la presencia del parque eólico como acción impactante, no tanto en el proceso de electrólisis.

En definitiva, los informes oficiales y los estudios de caso analizados destacan un bajo impacto ambiental para la producción de hidrógeno verde. Sin embargo, no se han encontrado estudios que ponderen de forma detallada los impactos acumulativos de la actividad en el uso de agua y suelo; en la flora, fauna y el patrimonio nacional; tampoco aspectos asociados a la participación ciudadana. Lógicamente, el riesgo ambiental resulta una preocupación siempre que existen actividades vinculadas a la explotación de los recursos hídricos. Más aún si la actividad se proyecta intensiva en regiones signadas por la escasez de este recurso, en ecosistemas frágiles como la estepa patagónica o la región altiplánica de la Puna.

Las lógicas de la metodología aplicada en los EIA sugieren que los impactos se pueden mitigar, prevenir, reducir y controlar a partir de la aplicación eficiente de un sistema de gestión ambiental. Pero para avanzar en este mercado, es preciso primero tener un claro conocimiento de las implicancias ambientales y elaborar una reglamentación coherente con estándares internacionales. Concretamente, se debe establecer los procedimientos formales que contribuyan a identificar los impactos ambientales y evaluar los efectos acumulativos y sinérgicos que puedan surgir de una política nacional orientada al hidrógeno verde. La redacción de una $E A E$, que acompañe la actualización del Régimen Legal de Promoción del Hidrógeno, sería un primer paso hacia ello. 


\section{Referencias bibliográficas}

Asociación Chilena de Hidrógeno. (2020). Misión Cavendish. Santiago, Chile.

Dincer, I. (2012). "Green methods for hydrogen production", Elsevier, International Journal of Hydrogen energy. Vol. 37.

Grün-und Umweltamt Mainz (2019). Energiepark Maiz zur elektrolytischen Erzeugung und zur Lagerung von Wasserstoff. Mainz.

Gutiérrez, J. L. (2005). El Hidrógeno, Combustible del Futuro, Real Academia de Ciencias Exactas, Físicas y Naturales, Vol. 99.

Harvey, D. (2014). Diecisiete contradicciones del capital y el fin del neoliberalismo. Madrid: Traficantes de Sueños.

Herrera, R. J. y Bonilla, M. (2009). Guía de evaluación ambiental estratégica. Santiago de Chile: Naciones Unidas, Santiago de Chile. Obtenido de http://repositorio. cepal. org/bitstream/handle/11362/3734/ S2009742_es. pdf.

Inodú (2020). Identificación de aspectos ambientales, sectoriales y territoriales para el desarrollo de proyectos de hidrógeno verde en toda su cadena de valor. Deutsche Gesellschaft für e Internationale Zusammenarbeit (GIZ) GmbH. Santiago, Chile.

IHS Markit (2020). Investment in green hydrogen production set to exceed $\$ 1$ billion USD by 2023. IHS Markit, Londres, Reino Unido. 03 de diciembre de 2020, <https:// news.ihsmarkit.com/prviewer/release_only/ slug/2020-12-03-investment-in-green-hydrogen-production-set-to-exceed-1-billionusd-by-2023>, 07 de enero de 2021.

International Finance Corporation -IFC(2015). Manual de Buena Práctica. Evaluación y Gestión de Impactos Acumulativos: Guía para el Sector Privado en Mercados Emergentes. Washington: WBG-IFC.

International Energy Agency -IEA- (2021). Net Zero by 2050. A roadmap for the Global Energy Sector. Disponible en: https://www. iea.órg/repórts/net-zeró-by-2050

International Energy Agency -IEA- (2019). The Future of Hydrogen. Seizing today's opportunities. International Energy Agency.
Ministerio de Energía (2020). Estrategia Nacional de Hidrógeno. Obtenido de Ministerio de Energía. Disponible en: https://www.energia.gob.cl/sites/default/files/mini-sitio/estrategianacional_hidrogeno-verde_vdef.pdf

Morante, J. R., Andreu, T., García, G., Guilera, J., Tarancón, A. y Torrell, M. (2020). "Hidrógeno. Vector energético de una economía descarbonizada". Fundación Naturgy, Madrid, España.

O' Connor, J. (2000). ¿Es posible el capitalismo sostenible?. Papeles de Población Vol. 6 N² 24. México: Universidad Autónoma del Estado de México Toluca.

Perez, A., Pérez, E., Dupraz, S., y Bolcich, J. (2016). Patagonia Wind-Hydrogen Project: Underground Storage and Methanation. 21st World Hydrogen Energy Conference, Jun 2016, Zaragoza, Spain.

Petroquímica (2020). Quieren reactivar la planta de hidrógeno de Pico Truncado. Revista Petroquímica. 30 de octubre de 2020, $<$ https://www.revistapetroquimica.com/ quieren-reactivar-la-planta-de-hidrogeno-depico-truncado/>, 10 de enero de 2021.

Regierungspräsidium Freiburg (2018). Bundes-Immissionsschutzgesetz (BImSchG) Power-to-GasAnlage in Grenzach-Wyhlen. Freiburg.

Rifkin, J. (2002). The hydrogen economy, the creation of the worldwide energy web and the redistribution of power on earth. Penguin Group, New York.

Rodríguez, C. R., Wuthrich, N., Cobos, J., Santa Cruz, R., Aisa, S., Jeandrevin, G., y Leiva, E. P. M. (2009). Aspectos económicos y ambientales de la producción de hidrógeno en la provincia de Córdoba, a partir de recursos eólicos. Avances en Energías Renovables y Medio Ambiente, 13.

Sanguinetti, A. (2020). El Gobierno busca impulsar el hidrógeno 'verde' como nuevo combustible en la Argentina, Instituto de Tecnologías del Hidrógeno y Energías Sostenibles, 27 de noviembre de 2020, <https:// ithes-uba.conicet.gov.ar/el-gobierno-busca- 
impulsar-el-hidrogeno-verde-como-nuevocombustible-en-la-argentina>, 07 de enero de 2021.

Webber, M. E. (2007). The water intensity of the transitional hydrogen economy. Environmental Research Letters, 2(3), 034007.

Winter, J. C. y Nitsch J. Eds (1988). Hydrogen as an Energy Carrier, Springer, Berlin.

\section{Notas}

1 Licenciado en Geografía y maestrando en Políticas Ambientales y Territoriales por la Universidad de Buenos Aires (UBA). Investigador del Instituto de Estudios de América Latina y el Caribe (IEALC-UBA) y becario por el Consejo Nacional de Investigaciones Científicas y Técnicas (CONICET) para el Doctorado en Geografía (UBA). Miembro del Grupo de Geopolítica y Bienes Comunes (GYBC).

2 Estados Unidos es el mayor productor, principalmente a partir de gas natural, destinado a la fabricación de amoníaco, refinamiento de petróleo, síntesis de metanol, entre otros (Inodú, 2020).

3 El desarrollo de las pruebas se llevó a cabo en distintas etapas que involucran ciclos de inyección y producción de gas natural e hidrógeno a distintos niveles de presión y concentración, con el objetivo de determinar la hermeticidad del reservorio (Pérez et al, 2016). 